Check for updates

Cite this: Phys. Chem. Chem. Phys., 2017, 19, 25853

Received 4th August 2017 Accepted 7th September 2017

DOI: $10.1039 / c 7 c p 01772 c$

rsc.li/pccp

\section{Acceleration of diffusion in ethylammonium nitrate ionic liquid confined between parallel glass plates $\dagger$}

\author{
Andrei Filippov, (D)*ab Oleg I. Gnezdilov, (D) ${ }^{\mathrm{b}}$ Nicklas Hjalmarsson, (D) ${ }^{\mathrm{c}}$ \\ Oleg N. Antzutkin, (DD a Sergei Glavatskih, (D) de István Furó (D ${ }^{f}$ and \\ Mark W. Rutland (D) ${ }^{\mathrm{cg}}$
}

\begin{abstract}
Diffusion of EAN confined between polar glass plates separated by a few micrometers is higher by a factor of ca. 2 as compared to bulk values. Formation of a new phase, different to the bulk, was suggested.
\end{abstract}

Ionic liquids (ILs) are molten salts formed typically of organic cations and either organic or inorganic anions. ${ }^{1,2}$ Their applications are continuously expanding, for example, as electrolyte materials in lithium batteries ${ }^{3}$ and ultracapacitors, ${ }^{4}$ as media for chemical reactions and separation, ${ }^{2,5}$ and as lubricants. ${ }^{6}$ Ethylammonium nitrate (EAN), synthesized by Paul Walden for the first time in $1914,{ }^{7}$ is the most studied protic $\mathrm{IL}^{1}{ }^{1}$ It is used as a replacement for organic solvents as a reaction medium, as a precipitating agent for protein crystallization, ${ }^{5}$ as an electrically conductive solvent in electrochemistry, ${ }^{3}$ amongst other applications. Similarly to water, EAN has a three-dimensional hydrogen-bonding network and can be used as an amphiphilic self-assembly medium. ${ }^{8}$ Bulk organization of EAN is often hypothesized as a bicontinuous, sponge-like structure. ${ }^{9-11}$

Recently, small-angle neutron scattering revealed that EAN itself exhibits an inherent amphiphilic nanostructure in the pure liquid state. ${ }^{9}$ For the lamellar structures deemed as most probable, the calculated Bragg spacing is approximately twice the ion-pair dimension of the IL, which suggests that the IL is

\footnotetext{
${ }^{a}$ Chemistry of Interfaces, Luleå University of Technology, SE-97187 Luleå, Sweden. E-mail: Andrei.Filippov@ltu.se; Tel: +46 73-6782225

${ }^{b}$ Institute of Physics, Kazan Federal University, 420008 Kazan, Russia

${ }^{c}$ Surface and Corrosion Science, KTH Royal Institute of Technology, SE-10044 Stockholm, Sweden

${ }^{d}$ System and Component Design, KTH Royal Institute of Technology, SE-10044 Stockholm, Sweden

${ }^{e}$ Department of Mechanical Construction and Production, Ghent University, B-9000 Ghent, Belgium

${ }^{f}$ Applied Physical Chemistry, KTH Royal Institute of Technology, SE-10044 Stockholm, Sweden

${ }^{g}$ RISE Technical Research Institute of Sweden, Chemistry Materials and Surfaces, Box 5607, SE-11486 Stockholm, Sweden

$\dagger$ Electronic supplementary information (ESI) available. See DOI: 10.1039/ c7cp01772c
}

structured on the length scale of the ions, with the (hydrogenous) alkyl groups associated together and segregated from the $\mathrm{H}$-bonded ionic moieties $-\mathrm{NH}_{3}{ }^{+}\left(\right.$or $\left.-\mathrm{ND}_{3}{ }^{+}\right)$and $\mathrm{NO}_{3}{ }^{-}$. Based on this finding, it has been suggested that surfaces may induce alignment of such locally ordered domains, creating a persistent molecular layering. ${ }^{10}$ Vibrational sum frequency spectroscopy and X-ray reflectivity studies ${ }^{11}$ confirmed the existence in EAN of significant interfacial structures within a $c a .3 \mathrm{~nm}$ thick layer. Surface structures have also been found in other ILs, ${ }^{12-14}$ sometimes persisting up to tens of nanometers into the bulk ${ }^{13,14}$ with a clear dependence on the molecular nature of the surface. Exceptionally extensive surface-induced structures with a thickness of up to $2 \mu \mathrm{m}$ were recently demonstrated for a number of imidazolium bis(trifluoromethylsulfonyl)imide ILs by Anaredy and Shaw. ${ }^{15}$ Much less attention has been paid to the structure and dynamics of ILs, which can be formed in a micrometer-scale confinement.

The purpose of this work was to study the dynamic features exhibited by EAN in the presence of polar surfaces and micrometer-scale confinement. For this purpose, we exploited NMR diffusion measurements, previously applied to a wide range of ILs. ${ }^{16-22}$ Of particular relevance, diffusion NMR has been used to study the molecular dynamics in ILs under confinement (coincidentally, all in silica pores). ${ }^{18,23-27}$ In comparison to NMR relaxation studies, diffusion NMR data are typically simpler to interpret and are more robust regarding experimental artefacts. ${ }^{28,29}$ Both simplicity and robustness are of great advantage for ILs with their frequently complex behaviour. In contrast to other transport methods, NMR diffusion data can be obtained selectively for different constituting moieties, in the present case the ethylammonium cation and the nitrate anion. This latter feature, as also shown here, can be aided by suitable isotope enrichment (deuterium, ${ }^{2} \mathrm{H}$, and ${ }^{15} \mathrm{~N}$ ).

EAN (see Fig. 1) consists of an ethylammonium (EA) cation and a nitrate (NI) anion. All samples were synthesized as described in the ESI, $\dagger$ following previously established protocols. ${ }^{30,31}$ While Sample 1 of EAN has a natural abundance (n.a.) of all isotopes: ${ }^{1} \mathrm{H}(99.98 \%),{ }^{13} \mathrm{C}(1.108 \%)$ and ${ }^{15} \mathrm{~N}(0.37 \%)$ 


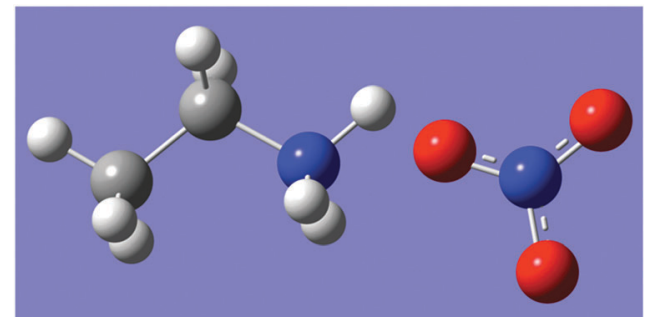

Fig. 1 The chemical structure of EAN consisting of an ethylammonium cation and a nitrate anion, with nitrogen (blue), oxygen (red), carbon (dark grey) and hydrogen (light grey) atoms shown.

in both EA and NI, the nitrate anion was ${ }^{15} \mathrm{~N}$ enriched $(\sim 98$ atom\%) in Sample 2 of EAN. Sample 3 of EAN was prepared by admixing $\mathrm{EAN}^{-\mathrm{D}_{5}}$, i.e. selectively deuterated at $\mathrm{CD}_{3}$ - and $\mathrm{CD}_{2}$-positions ( $\sim 98$ atom $\%$ of ${ }^{2} \mathrm{H}$ ) of the cation, with Sample 1 at a composition of $20 / 80$ mol\% of EAN-D $/$ EAN (n.a.). Prior to experiments, each sample was degassed under vacuum (at a pressure of less than $10^{-3}$ mbar and temperature of $313 \mathrm{~K}$ ) for 60 hours to remove absorbed water and gases. The chemical composition of each sample (including impurities) was established by liquid ${ }^{1} \mathrm{H}$ and ${ }^{13} \mathrm{C}$ NMR and is presented in the ESI. $\dagger$ In summary, the hydrogen and carbon contents associated with the ethylammonium cation were approximately 99\% in Sample 1 and more than $99.6 \%$ in Sample 2.

In a series of experiments, samples of EAN were confined between thin glass plates arranged in stacks (Fig. S1, ESI $\dagger$ ). The plates used $(5 \times 14 \times 0.1 \mathrm{~mm}$, Thermo Scientific Menzel Gläser, Menzel GmbH, Germany) were cleaned carefully before being used (see the ESI $\dagger$ ). Contact angle measurements with Milli-Q water provided a contact angle near $0^{\circ}$ (see the ESI $\dagger$ ), indicating hydrophilic glass surfaces. A stack of glass plates filled with EAN was prepared in a glove box in a dry nitrogen atmosphere. A drop of EAN was placed on the first plate, then it was covered by the second plate by placing another drop of EAN on the top, and so on until the thickness of the stack reached approximately $5 \mathrm{~mm}$. Excess of EAN from the sides of the stack was removed by wiping. The plates were thereafter placed in a sample cell of square cross section. The mean distance (spacing) between the glass plates was estimated by weighing the introduced EAN (see the ESI $\dagger$ ), which yielded $d \sim 3.8-4.5 \mu \mathrm{m}$. Measuring the thickness directly and subtracting it from the total glass thickness (see the ESI $\dagger$ ) indicates that this is a consistent value.

The ${ }^{1} \mathrm{H}$ (Samples 1 and 2) and ${ }^{15} \mathrm{~N}$ (Sample 2) NMR selfdiffusion measurements of EAN in the bulk were performed using a Pulsed-Field-Gradient (PFG) probe Diff50 (Bruker), and a Bruker Ascend Aeon WB 400 (Bruker BioSpin AG, Fällanden, Switzerland) NMR spectrometer was used with working frequencies of $400.27 \mathrm{MHz}$ for ${ }^{1} \mathrm{H}$ and $40.56 \mathrm{MHz}$ for ${ }^{15} \mathrm{~N}$. Data were processed using the Bruker TopSpin 3.5 software. Bulk samples of EAN (approximately $300 \mu \mathrm{l}$ each) were placed in a standard $5 \mathrm{~mm}$ glass NMR sample tube and sealed with a plastic cap to avoid contact with air. Before each measurement, the sample was equilibrated at the specified temperature for $20 \mathrm{~min}$.
The ${ }^{2} \mathrm{H}$ NMR spectra of Sample 3 were obtained using the same spectrometer with the quadrupole-echo pulse sequence.

The ${ }^{1} \mathrm{H}$ NMR diffusion measurements of EAN confined in the glass stack were performed using a NMR goniometer probe, which was used to macroscopically align the films in the sample stack at angles $0^{\circ}$ and $90^{\circ}$ with respect to the applied magnetic field gradient, the latter being co-parallel to the main magnetic field.

The diffusional decays (DDs) were recorded using the stimulated echo pulse sequence. For single-component isotropic diffusion, the signal intensity $A$ changes as: ${ }^{32,33}$

$$
A\left(\tau, \tau_{1}, g, \delta\right) \propto \exp \left(-\frac{2 \tau}{T_{2}}-\frac{\tau_{1}}{T_{1}}\right) \exp \left(-\gamma^{2} \delta^{2} g^{2} D t_{\mathrm{d}}\right)
$$

where $T_{1}$ and $T_{2}$ are the spin-lattice and the spin-spin relaxation times, respectively; $\tau$ and $\tau_{1}$ are time intervals in the pulse sequence; $\gamma$ is the gyromagnetic ratio for a used nucleus; $g$ and $\delta$ are the amplitude and the duration of the gradient pulse; $t_{\mathrm{d}}=(\Delta-\delta / 3)$ is the diffusion time; $\Delta=\left(\tau+\tau_{1}\right)$; and $D$ is the selfdiffusion coefficient. In the experiments, the gradient strength $g$ was varied with all other parameters kept constant. Some experiments were performed with varying $\tau$ and no effect on the DDs was observed. If not stated otherwise, the $D$ values were obtained by fitting eqn (1) to the experimental decays.

The anion lacks hydrogen and carbon. Hence the ${ }^{1} \mathrm{H}$ and ${ }^{13} \mathrm{C}$ NMR spectra of EAN arise exclusively from the EA cation (Fig. S2-S5 in the ESI $\dagger$ ). These spectra demonstrated three resonance lines, which were assigned to the $\mathrm{NH}_{3}{ }^{+}, \mathrm{CH}_{2}$ and $\mathrm{CH}_{3}$ moieties, in accordance with previously published data. ${ }^{34}$ As expected, the ${ }^{15} \mathrm{~N}$ NMR spectrum of the nitrate anion in Sample 2 revealed a single broad resonance line (Fig. $\mathrm{S} 6$ in the ESI $\dagger$ ) at $c a .380 \mathrm{ppm}$, in accordance with literature data. ${ }^{35}$

The DDs of the ${ }^{1} \mathrm{H}$ signals of the EA cation in Sample 1 (Fig. S7, ESI $\dagger$ ) and Sample 2, as well as the DD of the ${ }^{15} \mathrm{~N}$ signal of the nitrate anion (Fig. S8, ESI $\dagger$ ), are all linear in the semilogarithmic scale. Therefore, the mobility of each ion can be described by a single diffusion coefficient, the values of which $(D)$ are tabulated in Table S1 (ESI $\dagger)$. The $D$ values of the EA cation in both these samples coincide; therefore, any slight difference in the composition as that for Samples 1 and 2 does not influence the cation translation dynamics. An important observation is that the $D$ values of the nitrate anion are higher by a factor of $\sim 1.7$ than those of the EA cation. This means that the diffusive motion of cations and anions in bulk EAN is not tightly coupled (that is, ion pairing is not dominant). In dense ionic systems, self-diffusion is influenced strongly by electrostatic interactions and is no longer defined solely, as in simple aprotic liquids built up by neutral molecules, by molecular size. $^{21}$ In EAN, hydrogen bonding, too, is a significant intermolecular force with concomitant solvophobicity. ${ }^{9}$ To exemplify this point, one should recall that the viscosity of EAN is $c a .30$ times higher than that of nitropropane or similar liquids. ${ }^{36,37}$ In addition, EAN exhibits a nano-scale order $^{9}$ instead of a random local structure. Hence, it is difficult to pinpoint a single dominant reason for the factor-1.7 difference. We note that in another IL with nano-scale ordering, one could find in magnitude a rather 


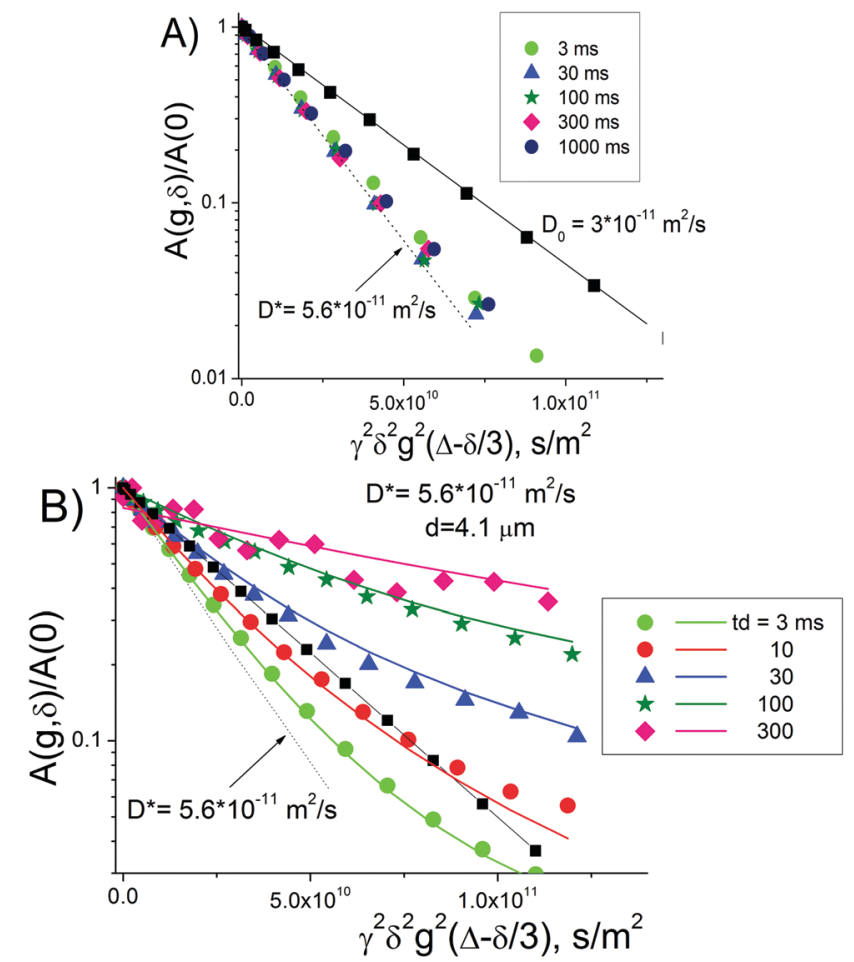

Fig. 2 The DDs of the ${ }^{1} \mathrm{H}$ NMR signals of the EA cation recorded at $293 \mathrm{~K}$ by pulsed-field-gradient stimulated echo experiments in bulk EAN (solid squares, $D_{0}=3.0 \times 10^{-11} \mathrm{~m}^{2} \mathrm{~s}^{-1}$ ) and in EAN films confined between parallel glass plates, with gradient directions along $(A)$ and perpendicular to (B) the plates. The DDs were obtained by increasing the gradient amplitude $g$, and the different colored symbols indicate data recorded at different diffusion times $t_{\mathrm{d}}$. Best fits (see the ESI $\dagger$ ) of suitable expressions for restricted diffusion to the data in (B) are presented by the lines of corresponding colors.

similar (factor-2) ratio between anion and cation diffusion coefficients. ${ }^{22}$ Yet, in that system, this ratio seemed to depend strongly on the nano-scale order that clearly shows the importance of local structure for the translational dynamics of the ions.

Fig. 2 shows the DDs of the ${ }^{1} \mathrm{H}$ signals of the EA cation with the field gradient directed along and normal to glass plates. Under current conditions, the DDs recorded are caused by diffusive molecular displacements in the IL films along and normal to the glass plates, respectively.

In the direction along the plates (Fig. 2A), the DDs maintain a linear form typical of the bulk state, but the slope (dotted line) of the decays and, consequently, the value of the derived self-diffusion coefficient $D_{\|}=D^{*}$ is a factor of $c a$. 1.9 higher than $D_{0}$ in the bulk (the decay for which is also shown for comparison). The DD is invariant to the changing diffusion time in the range of 50-1000 ms.

In the direction normal to the plates (Fig. 2B, colored symbols) and short diffusion times ( 3 and $10 \mathrm{~ms}$ ), the signal decays faster than that in bulk (black squares). The initial slopes of the DDs for these short diffusion times correspond to $D^{*}$. This means that the diffusion is accelerated to a similar extent along as well as normal to the plates, $D_{\perp}=D_{\|}=D^{*}$.

The forms of DDs (Fig. 2B) deviate from the singleexponential behavior typical for the bulk (recall eqn (1), shown here as a solid line and black squares), and the extent of this deviation increases as the diffusion time increases from $3 \mathrm{~ms}$ to $1 \mathrm{~s}$. The slopes of these DDs decrease with an increase in diffusion time, revealing that molecular displacements across the IL films confined between glass plates are hindered. ${ }^{28,32}$ The hindrance is constituted by the glass plates, whose separation of $c a .4 \mu \mathrm{m}$ is comparable to the diffusional path-length of ions in EAN, $L \approx \sqrt{ }\left(2 D^{*} \cdot t_{\mathrm{d}}\right),{ }^{38}$ which is, in the current case, between 1.8 and $5.8 \mu \mathrm{m}$ for $t_{\mathrm{d}}$ ranging from 30 to $300 \mathrm{~ms}$, respectively. As is well known, in such situations the mean displacement levels off, while the apparent mean diffusion coefficient decreases with increasing $t_{\mathrm{d}}$ revealing the same qualitative behavior as shown in Fig. 2B. Planar restriction (diffusion along the plane normal between parallel plates) is one of the simplest regular geometries, for which the DD of the confined liquids has been analytically solved, ${ }^{39}$ under the assumption of elastic collisions of diffusing molecules with the wall. Fits of that theoretical expression under assumptions concerning the distribution of plate-plate separation in the prepared sample stack (see Fig. S9 in the ESI $\dagger$ ) provided a mean distance between planes of $4.1 \mu \mathrm{m}$ that agrees rather well with the experimentally estimated value of $\sim 3.8 \mu \mathrm{m}$. In summary, the data in Fig. 2B are well explained by simple mechanical hindrance exerted by the glass plates on the diffusive displacement of cations (with $D^{*}$ ) in the IL film.

The DDs in Fig. 2A that arise from diffusive displacements in the IL films along the plates exhibit no dependence on the diffusion time and are thereby not influenced by any restrictions. This feature is as expected for free diffusion of ions along the IL films. Yet, in remarkable contrast, diffusion along the plates seems to be characterized by a diffusion coefficient $D_{\|}=D^{*}$ that is higher by a factor of $\sim 1.9$ than the value of $D_{0}$ observed in the bulk. This increase is so large that it is comparable to that obtained in the bulk by increasing the temperature by $c a .15 \mathrm{~K}$.

${ }^{15} \mathrm{~N}$ NMR diffusion measurements of EAN confined between plates were performed in order to investigate the effect of confinement on the diffusion of the nitrate anion. However, weak signals were obtained due to the lower filling factor of EAN between the plates as compared to the bulk sample (the ratio of the filling factors was estimated to be 0.001). The gyromagnetic ratio of ${ }^{15} \mathrm{~N}$ is lower than that of ${ }^{1} \mathrm{H}$ by a factor of 10 , while the number of ${ }^{15} \mathrm{~N}$ spins in EAN is lower than the number of protons by a factor of 8 . This is the main reason that we could measure diffusion of the cation $\left({ }^{1} \mathrm{H}\right)$ but not of the anion $\left({ }^{15} \mathrm{~N}\right)$.

In addition to PFG NMR experiments, the $T_{1}$ and $T_{2}$ NMR relaxation times of protons were also measured for the bulk and confined EAN (see the ESI $\dagger$ for details). Remarkably, the transverse relaxation time $T_{2}$ of $-\mathrm{NH}_{3}$ protons in EA cations in the confinement between glass plates is shorter than that of EA cations in the bulk by a factor of $c a .22$ (see Fig. S10 of the ESI $\dagger$ ), which can be explained, for example, by a significant acceleration in the exchange of $\mathrm{NH}_{3}$ protons in the whole layer of EAN confined between polar glass plates. Note that the presence of Si-OH and $\mathrm{H}_{2} \mathrm{O}$ protons at glass surfaces can only partly be invoked in the model explaining such a dramatic change in $T_{2}$, 
since the vast amount of EAN ions is located tens to hundreds of ionic radii away from the surfaces.

After having made these remarkable observations, it became imperative to consider and rule out possible experimental artifacts. Hence, the experimental set-ups and samples were carefully tested. Particular attention was paid to the possible effect of water; since the stack contained many glass plates, one could assume that water adsorbed at the glass surface may have dissolved in the IL films and contributed to faster molecular dynamics. To exclude this as a significant contribution, the diffusion coefficient in bulk EAN with increasing water content has been measured (see Fig. S11-S13 in the ESI $\dagger$ ). From these data and from the ${ }^{1} \mathrm{H}$ NMR spectra, from which the approximate water content in EAN could be estimated, we can conclude that the effect of water in the observed phenomenon is negligible (approximately $8 \mathrm{wt} \%$ water would be required to modify the diffusivity to the required extent, whereas the water content was shown to be $<0.1 \mathrm{wt} \%)$. Another possible source of artefact could be a capillary force, which might force the liquid to flow in narrow spaces. However, the samples were prepared some time before performing the experiments; therefore it seems unlikely that the experiments were not performed under equilibrium conditions. The only source of molecular motion was the thermal energy. Gravity might also lead to the flow in the thin layer of a liquid. In this case, the effect would depend on the orientation of the stack of glass plates relative to the gravity field of the Earth. No effects of orientation on the measured diffusion coefficient were observed. In summary, we conclude that the observed acceleration of diffusion between the confining glass plates is not an artifact, but an utterly surprising molecular (or structural) effect. This may affect not only the diffusion of EAN but also other physical parameters such as density. In the following we provide, with the support of some additional data, a tentative explanation to this observation.

Previous studies have established the notion that some surfaces can exert an effect on ILs that permeate the bulk for tens of nanometers. This effect was typically described as an imposed molecular order. ${ }^{9,11-15}$ Other studies have shown that changes in short-range molecular order can have an influence on the macroscopic (displacements over micrometers) selfdiffusion of the cations and anions in ILs. ${ }^{22}$ On this basis, we provide a tentative interpretation of our findings.

The diffusion path length over $3 \mathrm{~ms}$ with $D_{0}=3 \times 10^{-11} \mathrm{~m}^{2} \mathrm{~s}^{-1}$, the bulk diffusion coefficient, is $\left(2 \cdot D_{0} \cdot t_{\mathrm{d}}\right)^{0.5} \sim 0.42 \mu \mathrm{m}$, or if we take $D^{*}=5.6 \times 10^{-11} \mathrm{~m}^{2} \mathrm{~s}^{-1}$, the diffusion path is $\sim 0.58 \mu \mathrm{m}$. However, the DDs obtained for diffusion along the plates at $3 \mathrm{~ms}$ are single-exponential and coincide with the DDs obtained at longer diffusion times (Fig. 2A). This indicates that EAN diffusion is the same as in the center of a layer and near the plates (at a proximity of $\sim 0.5 \mu \mathrm{m}$ to the plates). Therefore, diffusion is accelerated not only in the thin layers near the surfaces, but in the whole thickness of the layer, even at the distance as far as $\sim 2 \mu \mathrm{m}$ from the surface.

Regarding the molecular order within the surface layer, if it is orientational akin to that exhibited by liquid crystals it may leave an imprint in NMR line splitting if quadrupolar nuclei, such as ${ }^{2} \mathrm{H}$, are used as an internal (in EA-D $_{5}$ or external $\left(\mathrm{D}_{2} \mathrm{O}\right.$ as a solute in EAN) spin-probe. This is the case if the structure within the layer is similar to that in liquid crystal phases with non-zero second-rank order parameter $S$, such as lamellar/smectic phases. In contrast, phases with $S=0$, such as cubic LCs, do not exhibit line splitting. To investigate this, we recorded the ${ }^{2} \mathrm{H}$ NMR spectra of Sample 3 prepared with EAN-D ${ }_{5}$. As shown by the NMR spectra (see Fig. S14 in the ESI $\dagger$ ), there is no detectable quadrupolar splitting in the ${ }^{2} \mathrm{H}$ NMR spectra. This finding suggests that either the surface layer is thin, in which case the ${ }^{2} \mathrm{H}$ NMR signal from it remains hidden in the background (at $\lambda=60 \mathrm{~nm}$, only $\sim 3 \%$ of the NMR signal arises from the surface layer), or that the molecular order is such that the order parameter $S$ remains zero.

The diffusivity of EA cations is clearly shown to be isotropic when confined between glass plates. The bulk diffusion properties are highly consistent with the literature consensus of a sponge-like self-assembly structure. Consideration of spin echo data, where no significant splitting is observed, further allows the conclusion to be drawn that the majority of cations are unaffected by the glass surfaces. Water is often present in ionic liquids and is fully compatible with EAN. In this work, it is categorically shown by exhaustive measurements that water associated with the polar glass plates cannot be solely responsible for the dramatic change in diffusivity compared to the bulk. At the same time, the confinement leads to acceleration in the exchange of $\mathrm{NH}_{3}$ protons in the whole layer. The most plausible idea for the accelerated diffusion of ions is that the nano-/microscale structural features of EAN confined between polar glass plates are considerably different from the bulk phase structure of EAN. We suggest that the mechanism of the increased rate of relaxation (decreased $T_{2}$ ) of $-\mathrm{NH}_{3}$ protons is an exchange process. Indeed, the maximum effect occurs only with "exchangeable" $-\mathrm{NH}_{3}$ protons; however, it is not the same with "non-exchangeable" $-\mathrm{CH}_{2}-$ and $-\mathrm{CH}_{3}$ protons. $-\mathrm{NH}_{3}$ protons are really generalized between $-\mathrm{NH}_{3}$ of the EA cation and the $\mathrm{NO}_{3}{ }^{-}$anion. In bulk EAN, they demonstrate a narrow line (as shown in Fig. S2 in the ESI $\dagger$ ). Therefore, they are in the condition of "fast exchange", where the difference in the chemical shift frequencies of the two sites is much less than the exchange rate. ${ }^{40}$ This is a case of the so-called "motional averaging" exchange regime. The acceleration of the rate of $T_{2}$ relaxation of $-\mathrm{NH}_{3}$ protons for EAN placed between polar glass plates (see Table S2 in the ESI $\dagger$ ) and broadening of the corresponding line in the ${ }^{1} \mathrm{H}$ NMR spectrum (Fig. S12 in the ESI $\dagger$ ) indicate that the exchange rate decreases and now $-\mathrm{NH}_{3}$ protons are in the "intermediate exchange" regime where the difference in the chemical shift frequencies of the two sites is of the order of the exchange rate. This transition from the "motional averaging" to the "intermediate" regime can be related to the suggested structural change of EAN occurring between polar glass plates. Indeed, in the spongelike phase of bulk EAN, the ions $-\mathrm{NH}_{3}{ }^{+}$and $\mathrm{NO}_{3}{ }^{-}$, as suggested, are located in a separate micro-phase, which is different from another micro-phase containing $-\mathrm{CH}_{2}-$ and $-\mathrm{CH}_{3}$ groups. This hinders diffusion but facilitates the proton exchange process. If the structure changes to a more 
homogeneous one, it might facilitate the diffusion of ions but will hinder proton exchange.

More work and other experimental techniques, such as the effect of surface roughness, surface polarity and variable hydrophobicity of the ionic liquid constituents, are needed to further clarify this remarkable phenomenon. Further experiments with different ionic liquids (firstly, ammonium nitrates), different substrates, different inter-plate gapes, without and with added solvents and salts would help to address the physics of the system.

\section{Conclusion}

In this work, we have experimentally shown that diffusion of ions in EAN confined between polar glass plates separated by a few micrometers is higher by a factor of $c a$. 1.9 as compared to self-diffusion of EAN ions in the bulk. After careful PFG NMR studies on EAN/water mixtures, we concluded that the most plausible suggestion for this observation is that the structural parameters of the whole sample of EAN in confinement are significantly different from those in bulk EAN. Having made these experimental observations, further experimental and theoretical studies are needed to fully understand this remarkable phenomenon. Given that the liquid properties are rather altered, these results have strong implications for interface intensive applications of ILs, such as lubricants and in electrochemical systems.

\section{Conflicts of interest}

There are no conflicts to declare.

\section{Acknowledgements}

The Knut and Alice Wallenberg foundation (project number KAW 2012.0078) and the Swedish Research Council (project numbers 621-2013-5171 (OA), 621-2012-3244 (IF), 621-2011-4600 and 621-2014-4694 (SG), and 621-2011-4361 (MR)) are gratefully acknowledged for their financial support. The Foundation in memory of J. C. and Seth M. Kempe and the laboratory fond at LTU are gratefully acknowledged for providing grants, from which a Bruker Aeon/Avance III NMR spectrometer at LTU has been purchased. NMR measurements were also partly carried out on the Bruker Avance III NMR spectrometer of the Federal Centre of Collective Facilities of the Kazan Federal University, Russia.

\section{References}

1 T. L. Greaves and C. J. Drummond, Chem. Rev., 2008, 108, 206.

2 R. D. Rogers and K. R. Seddon, Science, 2003, 302, 792.

3 C. Zhao, G. T. Burrell, A. A. J. Torriero, F. Separovic, N. F. Dunlop, D. R. MacFarlane and A. M. Bond, J. Phys. Chem. B, 2008, 113, 6923.

4 M. Lazzari, M. Mastragostino and F. Soavi, Electrochem. Commun., 2007, 9, 1567.
5 J. A. Garlitz, C. A. Summers, R. A. Flowers II and G. E. O. Borgstahl, Acta Crystallogr., Sect. D: Biol. Crystallogr., 1999, 55, 2037.

6 F. U. Shah, S. Glavatskih and O. N. Antzutkin, Tribol. Lett., 2013, 51, 281.

7 P. Walden, Bull. Acad. Imp. Sci. St.-Petersbourg, 1914, 8, 405.

8 H. J. Jiang, P. A. FitzGerald, A. Dolan, R. Atkin and G. G. Warr, J. Phys. Chem. B, 2014, 118, 9983.

9 R. Atkin and G. G. Warr, J. Phys. Chem. B, 2008, 112, 4164.

10 R. Atkin and G. G. Warr, J. Phys. Chem. C, 2007, 111, 5162.

11 P. Niga, D. Wakeham, A. Nelson, G. G. Warr, M. Rutland and R. Atkin, Langmuir, 2010, 26, 8282.

12 E. Sloutskin, B. M. Ocko, L. Tamam, I. Kuzmenko, T. Gog and M. Deutsch, J. Am. Chem. Soc., 2005, 21, 7796.

13 S. Bovio, A. Podesta, C. Lenardi and P. Milani, J. Phys. Chem. $B, 2009,113,6600$.

14 L. A. Jurado, H. Kim, A. Arcifa, A. Rossi, C. Leal, N. D. Spencer and R. M. Espinosa-Marzal, Phys. Chem. Chem. Phys., 2015, 17, 13613.

15 R. S. Anaredy and S. K. Shaw, Langmuir, 2016, 24, 5147.

16 M. Taher, F. U. Shah, A. Filippov, P. de Baets, S. Glavatskih and O. N. Antzutkin, RSC Adv., 2014, 4, 30617.

17 A. Filippov, F. U. Shah, M. Taher, S. Glavatskih and O. N. Antzutkin, Phys. Chem. Chem. Phys., 2013, 15, 9281.

18 A. Filippov, N. Azancheev, F. U. Shah, S. Glavatskih and O. N. Antzutkin, Microporous Mesoporous Mater., 2016, 230, 128.

19 R. Giernoth, Top. Curr. Chem., 2010, 290, 263.

$20 \mathrm{H}$. Li, in Structures and Interactions of Ionic Liquids, ed. S. Zhang, J. Wang, Q. Zhao and Q. Zhou, Structure and Bonding, Springer, 2014, vol. 151, p. 175.

21 H. Tokuda, K. Hayamizu, K. Ishii, A. B. H. Susan and M. Watanabe, J. Phys. Chem. B, 2005, 109, 6103.

22 A. E. Frise, T. Ichikawa, M. Yoshio, H. Ohno, S. V. Dvinskikh, T. Kato and I. Furó, Chem. Commun., 2010, 46, 728.

23 C. Iacob, J. R. Sangoro, P. Papadopoulus, T. Schubert, S. Naumov, R. Valiullin, J. Kärger and F. Kremer, Phys. Chem. Chem. Phys., 2010, 12, 13798.

24 E. D. Hazelbaker, R. Guillet-Nicolas, M. Thommes, F. Kleitz and S. Vasenkov, Microporous Mesoporous Mater., 2015, 206, 177.

25 M. Nayeri, M. T. Aronson, D. Bernin, B. F. Chmelka and A. Martinelli, Soft Matter, 2014, 10, 5618.

26 K. S. Han, X. Wang, S. Dai and E. W. Hagaman, J. Phys. Chem. C, 2013, 117, 15754.

27 T. Echelmeyer, H. W. Meyer and L. van Wüllen, Chem. Mater., 2009, 21, 2280.

28 W. S. Price, NMR Studies of Translational Motion: Principles and Applications, Cambridge University Press, Cambridge, 2009.

29 H. Walderhaug, O. Söderman and D. Topgaard, Prog. Nucl. Magn. Reson. Spectrosc., 2010, 56, 406.

30 D. F. Evans, A. Yamauchi, R. Roman and E. Z. Casassa, J. Colloid Interface Sci., 1982, 88, 89.

31 C. F. Poole, B. R. Kersten, S. S. J. Ho, M. E. Coddens and K. G. Furton, J. Chromatogr. A, 1986, 352, 407.

32 P. T. Callaghan, Principles of Nuclear Magnetic Resonance Microscopy, Clarendon, Oxford, 1991. 
33 J. E. Tanner, J. Chem. Phys., 1970, 52, 2523.

34 J. C. Thater, V. Gerard and C. Stubenrauch, Langmuir, 2014, 30, 8283.

35 F. A. Bovey, Nuclear Magnetic Resonance Spectroscopy, Academic Press, New York, 1988.

36 J. A. Riddick, W. B. Bunger and T. K. Sakano, Organic Solvents, John Wiley and Sons, New York, 4th edn, 1985, vol. II, p. 578.
37 J. A. Smith, G. B. Webber, G. G. Warr and R. Atkin, J. Phys. Chem. B, 2013, 117, 13930.

38 P. T. Callaghan and J. Stepisnik, in Advances in Magnetic and Optical Resonance, ed. W. S. Warren, Academic Press, San Diego, 1996, vol. 19, pp. 326-389.

39 P. Linse and O. Söderman, J. Magn. Reson., Ser. A, 1995, 116, 77.

40 M. Levitt, Spin Dynamics. Basics of Nuclear Magnetic Resonance, Wiley \& Sons, New York, 2nd edn, 2008. 